\title{
Anti-aging Effect on Skin of Autologous Transplantation of Tissue Fragments from Thawed Cryopreserved Ovaries
}

\author{
Hiroharu Imanishi ${ }^{1}$, Suguru Igarashi $^{1}$, Yoko Yamaguchi ${ }^{2}$, and Nao Suzuki ${ }^{1}$
}

(Received for Publication: April 27, 2018)

\begin{abstract}
Transplanting the ovaries of young mice into menopausal mice has been shown to extend their lifespan, suggesting that the reproductive organs may play an important role in combating aging. Preventing skin aging is an extremely important matter with respect to maintaining quality of life, but little basic research has been conducted to examine this issue. The effects of treatment with cryopreserved ovarian tissue, tissue hormone therapy (THT), and hormone replacement therapy on inhibiting skin aging were investigated in experimental animals. The effects on skin elasticity (R7) and body weight changes in 6-week-old mice resulting from the transplantation of cryopreserved ovarian tissue were evaluated, as were the effects on skin of estrogen administration after bilateral oophorectomy or transplantation. After the ovaries of 6-week-old mice were removed, the mice increased in weight over time. Their R7 temporarily decreased significantly to 3-week after oophorectomy, and subsequently improved. Mice were allocated into several different groups, including one that was given estrogen continuously for three weeks from immediately after oophorectomy and another that underwent transplantation of thawed ovarian tissue on the day after oophorectomy. While there was no difference of R7 between the hormone replaced groups and the sham surgery group, it was significantly lower in the non-replaced groups and the transplantation group than in the sham surgery group on day 14 and 18. R7 was still significantly lower on day 21 in the non-replaced group, but not in the transplantation group.R7 on day 21 in the transplantation group was significantly improved from that on day 18 . These results suggest that THT and hormone replacement therapy (HRT) might be equally effective. If techniques for THT using cryopreserved ovaries to maintain the blood estrogen concentration above a specific level can be established, this might help prevent or improve the deterioration of skin appearance in young women who require oophorectomy due to gynecological disease and also in childhood, adolescent, and young adult cancer patients.
\end{abstract}

\section{Key words}

Tissue hormone therapy, cryopreservation of ovarian tissue, mouse, ovarian transplantation, skin

\section{Introduction}

Women who have had an ovary removed at a young age are at increased risk of cognitive impairment or dementia, Parkinson's disease, and death from a neuropsychiatric disorder ${ }^{1)}$. Conversely, transplanting the ovaries of young mice into menopausal mice has extended their lifespan ${ }^{2}$, suggesting that the reproductive organs may play an important role in the appearance of disorders, control of the lifespan of liv- ing organisms, and combating aging.

Hormone replacement therapy (HRT) is an effective treatment for a range of symptoms that appear during the menopausal period, and it has also been found to have a cosmetic effect by improving skin elasticity and increasing collagen ${ }^{3 / 4)}$.

Preventing skin aging by suppressing the wrinkling and bagginess of the skin that appear with increasing age is an extremely important matter with respect to maintaining quality of life not only for

1 Departments of Obstetrics and Gynecology, St. Marianna University School of Medicine, Kawasaki, Japan

2 NANOEGG Research Laboratories, Inc., Tokyo, Japan 
menopausal women, but also for cancer survivors with primary ovarian insufficiency after chemotherapy or radiotherapy. However, little basic research has been carried out on this issue.

Therefore, the anti-aging effects on the skin of experimental animals treated with cryopreserved ovarian tissue, tissue hormone therapy (THT), and HRT were investigated.

\section{Experimental Procedures}

\section{Experimental Animals}

Six-week-old female Hos:HR-1 mice (Hoshino Laboratory Animals, Inc., Bando, Japan) were used. The mice were housed in a room of the St. Marianna University School of Medicine animal care unit under conditions of constant temperature $\left(23 \pm 1^{\circ} \mathrm{C}\right)$, constant humidity $(55 \% \pm 5 \%)$, and daylight $(6: 00-$ 18:00). They were fed commercial chow and given tap water to drink. Oophorectomy and transplantation were carried out under inhalation anesthesia. All experiments were approved by the Animal Care and Use Committee of the Animal Care Unit of St. Marianna University School of Medicine (Approval number: 1510009).

\section{Freezing, thawing, and transplantation of ova- ries}

Ovaries were frozen and thawed using the vitrification technique ${ }^{5)}$. The harvested ovaries were then finely sliced into eight pieces and placed in phosphate buffer I (PBI) medium containing 1M dimethyl sulfoxide (room temperature) ${ }^{6}$, after which they were placed into cryotubes (Nalge Nunc International KK, Yokohama, Japan). These were placed on ice for 5 min, after which $95 \mu \mathrm{L}$ of DAP 213 solution (2M dimethyl sulfoxide, $1 \mathrm{M}$ acetamide, $3 \mathrm{M}$ propylene glycol in PBI) maintained at $0^{\circ} \mathrm{C}$ were added to each cryotube. The tubes were again placed on ice for 5 minutes and then in liquid nitrogen for cryopreservation. Thawing was carried out by removing the cryotubes from the liquid nitrogen and allowing them to thaw at room temperature for 30 seconds, after which they were placed in dilution fluid consisting of PBI medium containing $0.25 \mathrm{M}$ sucrose $\left(37^{\circ} \mathrm{C}\right)$. After washing with PBI medium, they were stored in Whitten medium at $0^{\circ} \mathrm{C}$ until transplantation. This method refers to the experimental method reported in 2003 that succeeded in obtaining cryopreservation of ovarian tissue offspring with reproducible results by cryopreservation of ovaries using DAP 213 preservation solution, followed by transplantation to recipients af- ter thawing ${ }^{7)}$.

\section{Anti-aging effect on skin of the transplantation of cryopreserved ovarian tissue}

Six-week-old mice were divided into Group A ( sham surgery, $n=5$ ), Group B (oophorectomy, n=5), and Group C (cryopreserved ovarian tissue transplantation, $n=5)$.

After oophorectomy or sham surgery, body weight, skin elasticity as assessed by R2 (the restoration ratio after extension), R6 (the ratio of viscoelasticity to elastic deformation), and R7 (the ratio of the elastic portion at retraction), and blood estrogen concentrations were measured every week for nine weeks. The skin of group A and group B was subjected to histological analysis (hematoxylin-eosin $[\mathrm{H}-$ E] staining and picrosirius red staining) 13 weeks after the start of the experiment.

In Group $\mathrm{C}$, the cryopreserved ovarian tissue was thawed, and subcutaneous autologous transplantation was performed 9 weeks after oophorectomy. The return of the estrus cycle was confirmed 1 week after the slices were transplanted.

\section{Effect of postmenopausal HRT on skin}

Ovaries were removed from 6-week-old mice, and $17 \beta$-estradiol (E2) was administered subcutaneously at $0 \mathrm{pg}, 250 \mathrm{pg}, 2500 \mathrm{pg}$, and 25,000 pg (each, $\mathrm{n}=2$ ) and intraperitoneally at $0 \mathrm{pg}$ and $25,000 \mathrm{pg}$ (each, $\mathrm{n}=2$ ) after 13 weeks $^{8}$, and continued for two weeks. Body weight and skin elasticity (R7) were measured every week.

\section{Effect of perimenopausal HRT or THT on skin}

Six-week-old mice were allocated to a bilateral oophorectomy group (OVX), a sham surgery group, and a control group, an estrogen administration group, a progesterone administration group, an estrogen plus progesterone administration group, and a cryopreserved ovarian tissue transplantation group. Thawing and transplantation of cryopreserved ovarian tissue were performed the day after oophorectomy (each group $\mathrm{n}=5$ ). Estrogen 25,000 pg and/or progesterone $1 \mathrm{mg}$ (Wako Pure Chemical Industries, Osaka, Japan) was administered intraperitoneally every 3-4 days for three weeks, and body weight and skin elasticity (R7) were measured over time. Both estrogen and progesterone were dissolved in olive oil (Wako Pure Chemical Industries, Osaka, Japan) before administration to the mice, and only the olive oil was administered to the control group. 
The skin of the mice of all groups was subjected to histological evaluation (H-E staining and picrosirius red staining) 3 weeks after the start of the experiment.

Data are expressed as mean \pm SD. Statistical analysis was performed using repeated two-way analysis of variance, with $\mathrm{p}<0.05$ considered the level of significance. Data analysis was conducted with the Statistical Package for Social Science (SPSS).

\section{Results}

At two weeks after oophorectomy, skin elasticity, measured in terms of R7 (the ratio of the elastic portion at retraction), was significantly decreased in Groups B (oophorectomy group) and C (cryopreserved ovarian tissue transplantation group), both of which had undergone oophorectomy, compared with those in Group A, which did not undergo oophorectomy (Figure 1). And at three weeks, R7 in group $\mathrm{C}$ was still significantly lower than in group A.

Body weight at seven, eight and nine weeks after oophorectomy and mean body weight for nine weeks were significantly greater in Groups B and C than in Group A $(\mathrm{A}: 27.8 \pm 0.5, \mathrm{~B}+\mathrm{C}: 32.2 \pm 0.4$, $\mathrm{p}=0.022$ ) (Table 1). Autopsies showed accumulation of visceral fat in the lower abdomen, particularly around the uterus, and a pronounced increase in fat near the rump in the mouse of group B (Figure 2a, b). Body weight was compared for four weeks after the thawing and transplantation of cryopreserved ovaries, but no significant differences were observed among the three groups (Table 2). Histological evaluation also found no significant differences between group A and group B in terms of the thickness of the dermal or muscle layers and collagen content (Figures $2 \mathbf{c}, \mathbf{d})$. The mean blood E2 concentration was 5 $\mathrm{pg} / \mathrm{mL}$ in Group A, much higher than in Groups B and $\mathrm{C}$ (Figure 3). The mean blood E2 concentration in groups $\mathrm{B}$ and $\mathrm{C}$ was $\leq 2 \mathrm{pg} / \mathrm{mL}$, which is lower than the detection sensitivity of the kit; therefore, the actual average E2 concentration was unknown.

Since in the initial experiment, significant differences were observed in body weight and in R7 (the ratio of the elastic portion at retraction), body weight and R7 were measured thereafter in the postmenopausal and perimenopausal therapy experiments. The postmenopausal experiment was first started with $n=$ 2 , but sufficient R7 data for analysis were not obtained, and the experiment and analysis were concluded prematurely (Figure 4).

In the perimenopausal experiment, body weight
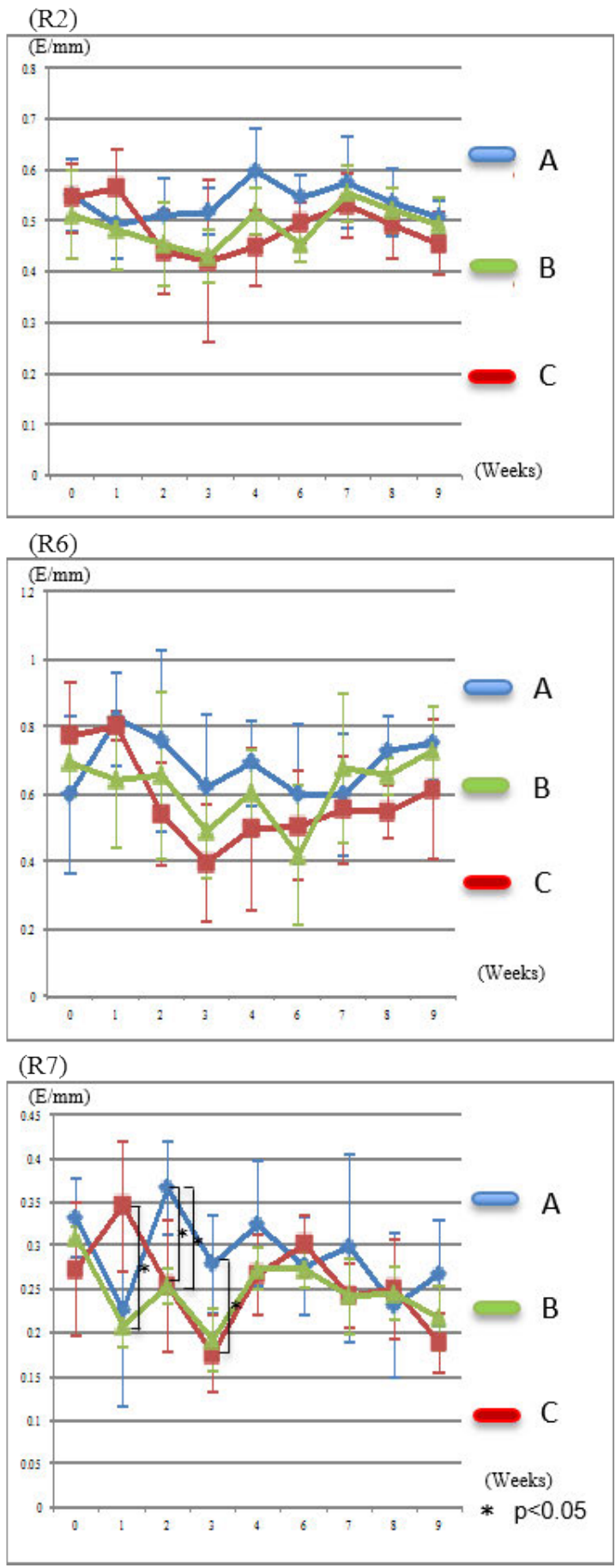

Figure 1. Changes of skin elasticity after oophorectomy for nine weeks.

and elasticity were evaluated for three weeks after the start of the experiment. Body weight increased over time in all groups, with no significant differences among the all groups (Figure 5a). 
Table 1. Average Weight Change before Cryopreserved Ovarian Tissue Transplantation

\begin{tabular}{|c|c|c|c|c|c|c|c|c|c|}
\hline Weeks/Groups & 1 & 2 & 3 & 4 & 5 & 6 & 7 & 8 & 9 \\
\hline Group A (g) & $19.1 \pm 1.1$ & $19.5 \pm 0.9$ & $20.6 \pm 0.9$ & $23.0 \pm 0.7$ & $24.1 \pm 0.6$ & $24.9 \pm 0.5$ & $25.4 \pm 0.6$ & $26.7 \pm 0.5$ & $27.8 \pm 0.5$ \\
\hline Group B+C (g) & $19.6 \pm 1.0$ & $20.0 \pm 0.6$ & $22.1 \pm 0.5$ & $24.7 \pm 0.5$ & $26.0 \pm 0.7$ & $27.9 \pm 0.5$ & $29.5 \pm 0.5$ & $31.0 \pm 0.3$ & $32.2 \pm 0.4$ \\
\hline
\end{tabular}

(a) Group A

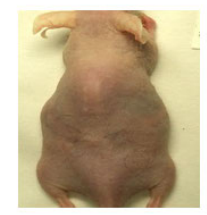

(c) Group A

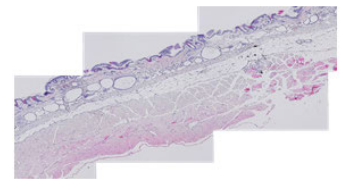

(b) Group B

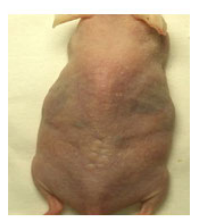

(d) Group B

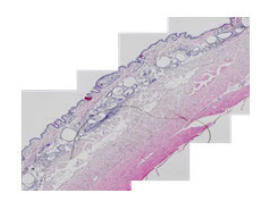

Figure 2. (a, b) Macroscopic findings of group A and group B at 13-week after oophorectomy. (c, d) Microscopic findings of group A and group B at 13-week after oophorectomy.

Table 2. Average Weight Change after Cryopreserved Ovarian Tissue Transplantation

\begin{tabular}{ccccc} 
Weeks/Groups & 1 & 2 & 3 & 4 \\
\hline 6w Group A (g) & $28.1 \pm 0.4$ & $28.7 \pm 0.5$ & $28.5 \pm 0.5$ & $29.4 \pm 0.5$ \\
6w Group B (g) & $32.3 \pm 0.5$ & $33.0 \pm 0.6$ & $33.5 \pm 0.6$ & $34.2 \pm 0.5$ \\
6w Group C (g) & $32.2 \pm 0.4$ & $32.7 \pm 0.5$ & $33.3 \pm 0.5$ & $33.9 \pm 0.7$
\end{tabular}

Regarding R7 change in each group as shown in Figure 5b, in the OVX group, there were significant differences between before the start of the experiment and Days 14, 18, and 21, and between Day 11 and Days 14, 18, and 21, with higher R7 before the start of the experiment and on Day 11, and R7 decreased on Days 14,18 , and 21. In the control group, there were significant differences between before the start of the experiment and Days 14 and 18, and between Day 11 and Days 14, 18, and 21. R7 was higher before the start of the experiment and on Day 11, and lower on Days 14, 18, and 21. In the progesterone group, there were significant differences between before the start of the experiment and Days 14 and 18,

and between Day 11 and Days 14 and 18. R7 was higher before the start of the experiment and on Day 11 than on Days 14 and 18. In the estrogen + progesterone group, there were significant differences between before the start of the experiment and Day 18 and between Day 11 and Day 18, and R7 was significantly higher before the start of the experiment and on Day 11 than on Day 18. In the transplantation group, there were significant differences between before the start of the experiment and Days 14 and 18, between Day 11 and Day 18, and between Day 18 and Day 21. R7 was higher before the experiment than on Days 14 and 18, higher on Day 11 than on Day 18, and also higher on Day 21 than on Day 18. R7 was thus lowest on Day 18, and the differences between this day and before the start of the experiment, Day 11, and Day 21 were all significant.

Regarding comparison of R7 among the all groups as shown in Figure 5c, on Day 14 and 18, there were significant differences between the OVX group and the sham surgery group and between the transplantation group and the sham surgery group. R7 was significantly higher in the sham surgery group than in either the OVX group and the transplantation group. On Day 21, still there was a significant difference between the OVX group and the sham surgery group, but not between the transplantation group and the sham surgery group. R7 in the groups with HRT was not different from the sham surgery groups during the experiment period.

\section{Discussion}

HRT is an effective treatment for a range of symptoms that appear during the menopausal period as a result of diminished ovarian function and is also effective against osteoporosis, fat metabolism, arteriosclerosis, diminished cognitive function, and depressed mood. However, it is known to increase the risk of several disorders including breast cancer $^{9}$, heart disease due to arteriovenous thromboembo$\operatorname{lism}^{10)}$, cerebrovascular disease ${ }^{11)}$, and exacerbation of liver dysfunction ${ }^{12)}$. 


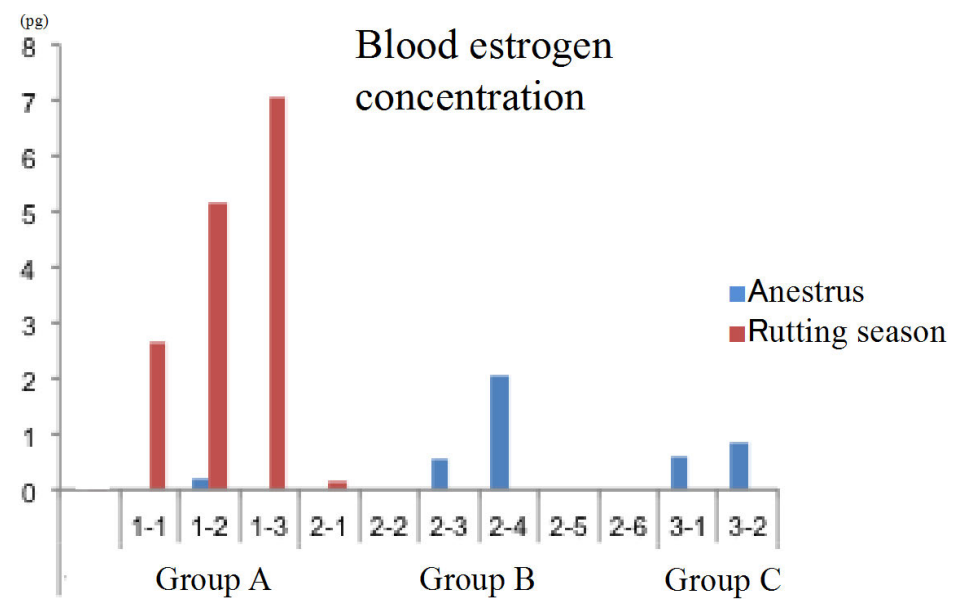

Figure 3. Results of measurements of estrogen blood concentration at 13week after oophorectomy (4-week after transplantation in group C).

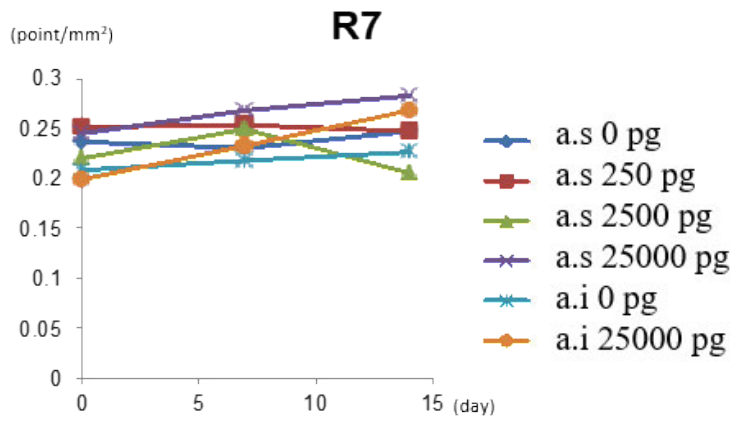

Figure 4. Changes of skin elasticity (R7) in each group of postmenopausal experiments.

In the field of reproductive medicine, the technology for cryopreservation of ovarian tissue with the aim of preserving fertility has made rapid strides since the beginning of the 21 st century, and there have been many reported cases of young cancer patients becoming pregnant and giving birth following treatment with anticancer drugs ${ }^{13) 14}$. The rate of becoming pregnant among women who underwent ovarian tissue transplantation was $35 \%{ }^{15)}$. In one recent case, a 9-year-old girl diagnosed as having Ewing's sarcoma had her ovaries cryopreserved prior to the start of chemotherapy and radiotherapy, and they were transplanted after treatment at age 13 years. Subsequently, female hormones were secreted by the transplanted ovaries and menarche occurred, but she became amenorrheic after approximately 6 months ${ }^{16)}$.

THT, comprising treatment with female hormones secreted by the patient's own transplanted ovaries for purposes other than becoming pregnant, is a promising new method of treatment ${ }^{17}$. Whether the transplantation of cryopreserved ovarian tissue has a cosmetic effect on skin equivalent to that of HRT was investigated using the ovarian tissue cryopreservation techniques developed via continuing basic studies in Macaca fascicularis and rats ${ }^{18-20)}$.

Previous studies have shown that visceral fat increases after oophorectomy due to the lack of female hormones ${ }^{21}$. In the present study, when the ovaries of young mice were removed, skin elasticity (R7) decreased temporarily to 3-week after oophorectomy, and then improved as shown in Figure 1. In reproductive medicine, the objective of ovarian tissue transplants is the growth and development of ovarian follicles after transplantation, and follicle-stimulating hormone (FSH) is administered extracorporeally ${ }^{18)}$. Follicle growth stimulates estrogen secretion, but since pregnancy was not the objective in the present study, FSH was not administered. The present results may have been partly due to the effect of growth hormone. Female hormones have been shown to improve the viscoelasticity of cutaneous connective tissue ${ }^{22}$, and the repeated administration of growth hormone may also provide protection against thinning of the skin in older people ${ }^{23}$. Growth hormone is also known to act to promote collagen synthesis and stimulate angiogenesis ${ }^{24)}$, and it has been shown to accelerate wound healing ${ }^{25}$. Because young mice were used in this study, there is a possibility that growth hormone influenced skin elasticity.

Given that future improvement of freezing and thawing techniques for the ovary and implantation sites will likely lead to the clinical use of THT using 
(a)
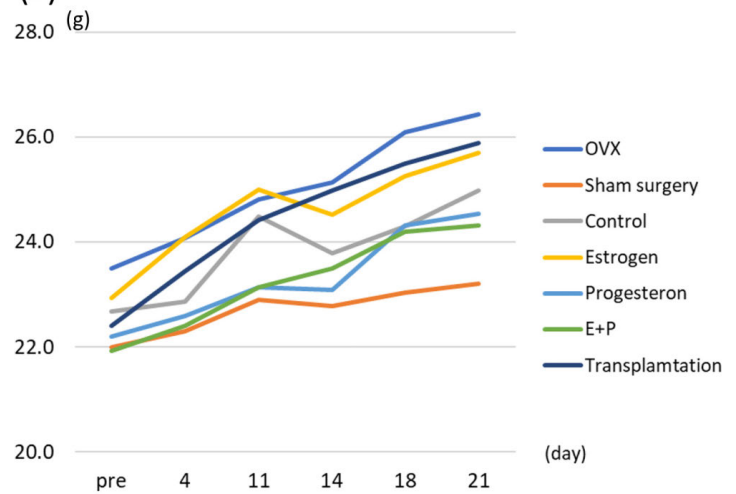

(b)

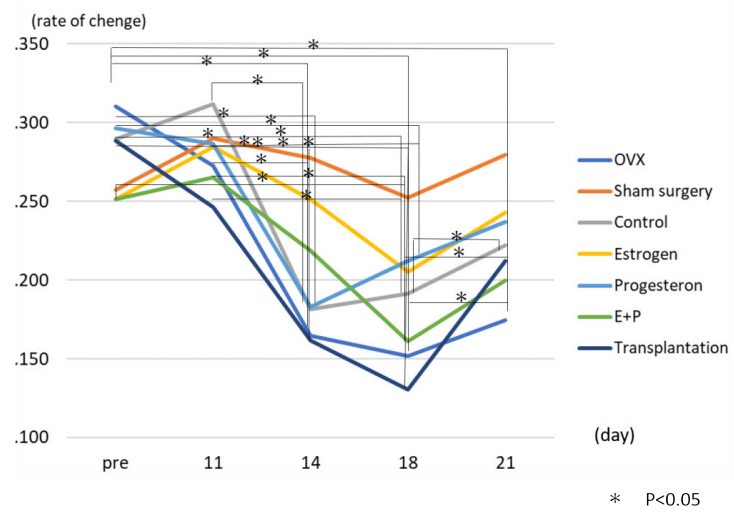

(c)

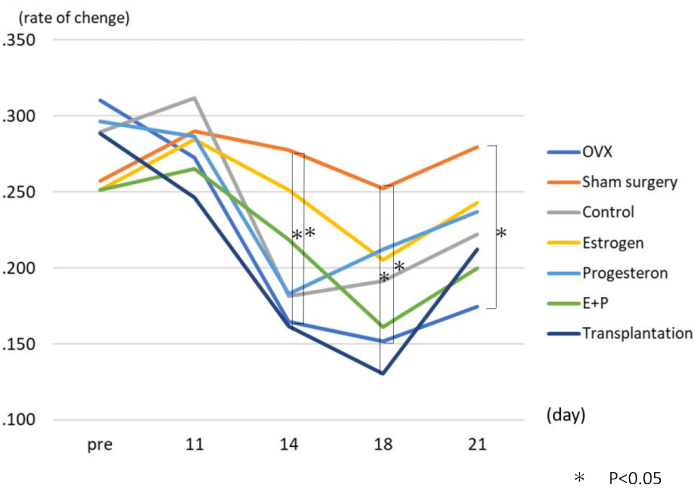

Figure 5. Effects of perimenopausal HRT and THT experiments on body weight and skin elasticity

(a) Changes of body weight over time in each group.

(b) Changes of skin elasticity (R7) in each group.

(c) Comparison of R7 among the groups.

ovarian tissue, an additional experiment was performed to investigate the effect of postmenopausal HRT on skin. Observations were carried out for two weeks after E2 administration. The experiment was initially started with $n=2$, but since sufficient body weight and skin elasticity data for analysis were not obtained from any of the groups, the experiment and analysis were concluded prematurely (Figure 4). The results suggested that, after oophorectomy in young mice, the administration of female hormones may have little effect on skin elasticity after a certain amount of time has elapsed.

Whether the administration of estrogen and other hormones at an early stage after oophorectomy and the transplantation of cryopreserved and thawed ovarian tissue would both temporarily inhibit the decline in skin elasticity and act to increase its elasticity was investigated. Whether the effect of ovarian tissue transplantation was equal to that of estrogen was also investigated. Body weight gradually increased over time in all groups, and there was no obvious significant difference.

Focusing on R7 (the ratio of the elastic portion at retraction), for which a significant difference was evident in the first experiment, multiple comparisons between the different groups at each time point after the simple main effect showed that, though skin elasticity was significantly lower in the transplantation group than the sham surgery group on Day 18, three days later, it had recovered to an equivalent level. The estrogen group also maintained equivalent elasticity to the sham surgery group, but it did not improve to higher level of the sham surgery group. However, the present results suggest that the effect of ovarian tissue transplantation on skin may become evident from around 3 weeks after transplantation (Figure $\mathbf{5 b}, \mathbf{c}$ ).

If HRT is started within 10 years after menopause, the risk of cardiovascular impairment is lower than if it is started more than 20 years after menopause ${ }^{26)}$, which suggests that if HRT is started at a comparatively early stage of estrogen deficiency, it may prevent the decrease in skin elasticity.

A distinctive feature of the results of this experiment was that skin elasticity was lowest on Day 18 in those groups that had undergone some sort of postoophorectomy treatment, such as intraperitoneal drug administration or transplantation, that is, those mice with estrogen in their bodies (the estrogen group, the estrogen + progesterone group, and the transplantation group), whereas in those groups that lacked estrogen (the control group and the progesterone group), it was lowest on day 14, 4 days earlier.

Estrogen is known to increase skin elasticity and its collagen content ${ }^{344)}$. The control group and the progesterone group both lacked estrogen, and so they 
should have followed the same course as the OVX group, with skin elasticity reaching its lowest value on around Day 18 and remaining low thereafter. However, in these two groups, elasticity started to recover earlier than in the groups either given estrogen or in which estrogen was present on Day 14 after oophorectomy. This may have been due to some sort of stimulation in the abdominal cavity in a no-estrogen environment, inducing a factor other than estrogen that affects skin, such as growth hormone ${ }^{22-25)}$, which immediately exerted an effect equivalent to that of estrogen (Figure 5b, c).

The present study has two limitations. First, it is not clear why E2 was not secreted from the transplanted ovaries, and whether the problem relates to the freezing method or the transplant site requires further investigation. Second, rodents were used in the experiments. It is difficult to draw comparisons between studies in rodents, which have a short menstrual cycle, and humans. Ideally, primates (monkeys) should have been used because their menstrual cycle is similar to that of humans. However, primates are expensive, and few institutions are equipped to look after them; thus, it was not possible to use them in the present study.

In the present study, after the ovaries of young mice were removed, skin elasticity decreased temporarily to 3-week after oophorectomy, but subsequently improved. Mice were allocated into several groups, including one that was given estrogen continuously from immediately after oophorectomy and another that underwent transplantation of thawed ovarian tissue on the day after oophorectomy. An investigation of whether this would prevent elasticity from decreasing soon after oophorectomy or even increase it found that, though elasticity was preserved in comparison with mice that underwent sham surgery, it did not increase significantly. These results suggest that perimenopausal THT and HRT might be equally effective. If techniques for THT using cryopreserved ovaries to maintain the blood estrogen concentration above a specific level can be established, this might help to prevent or improve the deterioration of skin appearance in young women who require oophorectomy due to gynecological disease and also in childhood, adolescent, and young adult cancer patients.

\section{Acknowledgments}

We thank Teruaki Nagasawa, Yoshiki Kubota, and Nanako Shimura of NANOEGG Research Labo- ratories, Inc. for helping with the research.

\section{Author's contributions}

Hiroharu Imanishi conducted the experiments, performed the analysis, and wrote the manuscript; Yoko Yamaguchi contributed intellectually to the experimental design and edited the manuscript; Suguru Igarashi contributed to data interpretation and manuscript writing; Nao Suzuki conceived the project, designed the experiments, and wrote the manuscript.

\section{References}

1) Yasui $T$, Hayashi $K$, Mizunuma $H$, Kubota $T$, Aso T, Matsumura Y, Lee JS, Suzuki S. Factors associated with premature ovarian failure, early menopause and earlier onset of menopause in Japanese women. Maturitas 2012; 72: 249-255.

2) Cargill SL, Carey JR, Müller HG, Anderson G. Age of ovary determines remaining life expectancy in old ovariectomized mice. Aging Cell 2003; 2: 185-190.

3) Ohta H, Makita K, Kawashima T, Kinoshita $S$, Takenouchi M, Nozawa S. Relationship between dermato-physiological changes and hormonal status in pre-, peri-, and postmenopausal women. Maturitas 1998; 30: 55-62.

4) Sumino $H$, Ichikawa $S$, Abe $M$, Endo $Y$, Ishikawa O, Kurabayashi M. Effects of aging, menopause, and hormone replacement therapy on forearm skin elasticity in women. J Am Geriatr Soc 2004; 52: 945-949.

5) Nakao K, Nakagata N, Katsuki M. Simple and efficient vitrification procedure for cryopreservation of mouse embryos. Exp Anim 1997; 46: 231-234.

6) Whittingham DG. Embryo banks in the future of developmental genetics. Genetics 1974; 78: 395-402.

7) Migishima F, Suzuki-Migishima R, Song SY, Kuramochi T, Azuma S, Nishijima M, Yokoyama M. Successful cryopreservation of mouse ovaries by vitrification. Biol Reprod 2003; 68: 881-887.

8) Suzuki A, Urushidani H, Watanabe H, Sato T, Iguchi T, Kobayashi T, Ohta Y. Comparison of estrogen responsive genes in the mouse uterus, vagina and mammary gland. $\mathrm{J}$ Vet Med Sci 2007; 69: 725-731.

9) Nelson HD, Humphrey LL, Nygren P, Teutsch SM, Allan JD. Post-menopausal hormone replacement therapy: scientific review. JAMA 
2002; 288: 872-881.

10) Rossouw JE, Anderson GL, Prentice RL, LaCroix AZ, Kooperberg C, Stefanick ML, Jackson RD, Beresford SA, Howard BV, Johnson KC, Kotchen JM, Ockene J; Writing Group for the Women's Health Initiative Investigators. Risks and benefits of estrogen plus progestin in healthy postmenopausal women. JAMA 2002; 288: 321-333.

11) Wassertheil-Smoller $S$, Hendrix $S$, Limacher $M$, Heiss G, Kooperberg C, Baird A, Kotchen T, Curb JD, Black H, Rossouw JE, Aragaki A, Safford M, Stein E, Laowattana S, Mysiw WJ; WHI Investigators. Effect of estrogen plus progestin on stroke in postmenopausal women. JAMA 2003; 289: 2673-2684.

12) Shoupe D. Contraindications to hormone replacement. In: Lobo RA, editor. Treatment of the postmenopausal women; Basic and clinical aspects. 2nd ed. Philadelphia: Lippincott Williams \& Wilkins; 1999; 567-574.

13) Donnez J, Dolmans MM, Demylle D, Jadoul P, Pirard C, Squifflet J, Martinez-Madrid B, van Langendonckt A. Livebirth after orthotopic transplantation of cryopreserved ovarian tissue. Lancet 2004: 346: 1405-1410.

14) Donnez J, Dolmans MM. Ovarian cortex transplantation; 60 reported live births brings the success and worldwide expansion of the technique towards routine clinical practice. J Assist Reprod Genet 2015; 32: 1167-1170.

15) Rodriguez-Wallberg KA, Tanbo T, Tinkanen H, Thurin-Kjellberg A, Nedstrand E, Kitlinski ML, Macklon KT, Ernst E, Fedder J, Tiitinen A, Morin-Papunen L, Einarsson S, Jokimaa V, Hippeläinen M, Lood M, Gudmundsson J, Olofsson JI, Andersen CY. Ovarian tissue cryopreservation and transplantation among alternatives for fertility preservation in the Nordic countries compilation of 20 years of multicenter experience. Acta Obstet Gynecol Scand 2016; 95: 1015-1026.

16) Ernst E, Kjærsgaard M, Birkebæk NH, Clausen $\mathrm{N}$, Andersen CY. Case report: Stimulation of puberty in a girl with chemo- and radiation therapy induced ovarian failure by transplantation of a small part of her frozen/thawed ovarian tissue. Eur J Cancer 2013: 49: 911-914.

17) von Wolff M, Stute P. Cryopreservation and transplantation of ovarian tissue exclusively to postpone menopause: technically possible but endocrinologically doubtful. Reprod Biomed Online 2015; 31: 718-721.

18) Igarashi $S$, Suzuki N, Hashimoto $S$, Takae $S$, Takenoshita M, Hosoi Y, Morimoto Y, Ishizuka B. Heterotopic autotransplantation of ovarian cortex in cynomolgus monkeys. Hum Cell 2010; 23: 26-34.

19) Igarashi S, Suzuki N, Osada M, Takae S, Tarumi W, Ishizuka B. Cryopreservation of ovarian tissue after pretreatment with a gonadotropin-releasing hormone agonist. Reprod Med Biol 2010; 9: 197-203.

20) Suzuki N, Hashimoto S, Igarashi S, Takae S, Yamanaka M, Yamochi T, Takenoshita M, Hosoi Y, Morimoto Y, Ishizuka B. Assessment of long-term function of heterotopic transplants of vitrified ovarian tissue in cynomolgus monkeys. Hum Reprod 2012; 27: 2420-2429.

21) Zoth N, Weigt C, Laudenbach-Leschowski U, Diel P. Physical activity and estrogen treatment reduce visceral body fat and serum levels of leptin in an additive manner in a diet induced animal model of obesity. J Steroid Biochem Mol Biol 2010; 122: 100-105.

22) Pierard GE, Hermanns-Le P, Paquet P, PiérardFranchimont C.Skin viscoelasticity during hormone replacement therapy for climacteric ageing. Int J Cosmet Sci 2014; 36: 88-92.

23) Rudman D, Feller AG, Nagraj HS, Gergans GA, Lalitha PY, Goldberg AF, Schlenker RA, Cohn L, Rudman IW, Mattson DE. Effects of human growth hormone in men over 60 years old. N Engl J Med 1990; 323: 1-6.

24) Kann P, Piepkorn B, Schehler B, Lotz J, Prellwitz W, Beyer J. Growth hormone substitution in growth hormone-deficient adults: Effects on collagen type I synthesis and thickness. Exp Clin Endocrinol Diabetes 1996; 104: 327-333.

25) Messias de Lima CF, deAraujo Vieira LF, de Carvalho Wanderley LA, de Souza Ferro JN, Smaniotto S. Topical growth hormone accelerates wound healing in mice. Wounds 2017; 29: 387-392.

26) Rossouw JE, Prentice RL, Manson JF, Wu L, Barad D, Barnabei VM, Ko M, LaCroix AZ, Margolis KL, Stefanick ML. Postmenopausal hormone therapy and risk of cardiovascular disease by age and years since menopause. JAMA 2007; 297: 1465-1477. 